\title{
Tratamento da tosse associada a infecções do trato respiratório superior em crianças: qual a melhor evidência?
}

Marlene Sousa*

\section{RESUMO}

Objectivos: Rever a evidência disponível sobre terapêutica da tosse associada a infecções do tracto respiratório superior em crianças entre seis e doze anos.

Fontes de Dados: Foram consultadas as bases de dados National Guideline Clearinghouse, Canadian Medical Association Infobase, Publeg, Cochrane Library, Dare, Trip Database, National Library of Guidelines Specialist Library, Pubmed e Índex das Revistas Médicas Portuguesas e revistas as referências bibliográficas dos artigos relevantes.

Métodos de Revisão: Foram pesquisadas as fontes de dados referidas, usando os termos MeSH child, cough, upper respiratory infection e therapeutic, e os respectivos DeCS, para obtenção de artigos escritos em Inglês, Espanhol, Francês ou Português publicados até Julho de 2008. Os estudos foram classificados usando a taxonomia Strenght of Recomendation Taxonomy (SORT).

Excluíram-se estudos discordantes com objectivo, estudos noutras faixas etárias e aqueles relativos a condições clínicas que não ITRS.

Resultados: Foram obtidos oitenta e dois estudos e seleccionados doze que cumpriam critérios de inclusão. Dos estudos analisados, nove mostraram que os anti-tússicos, os anti-histamínicos e as combinações anti-histamínico/descongestionante são ineficazes no tratamento da tosse. Outros dois, analisando uma combinação de anti-tússico e broncodilatador e um broncodilatador isolado mostraram ineficácia destas substâncias no tratamento da tosse e, noutro, não houve benefício no aumento da ingestão oral de fluidos. Dois estudos mostraram melhoria sintomática não significativa do sintoma com a utilização de xaropes pediátricos para a tosse. Para expectorantes e metilxantinas não foram encontrados estudos em crianças.

Conclusões: Não há evidência da eficácia do uso de anti-tússicos, expectorantes, anti-histamínicos e hidratação oral abundante para o tratamento da tosse (SOR A). Não há evidência da eficácia dos broncodilatadores na diminuição deste sintoma (SOR B). Xaropes pediátricos usados no tratamento a tosse têm efeito pouco significativo na diminuição da mesma (SOR B). São necessários mais estudos aleatorizados controlados de boa qualidade acerca da terapêutica da tosse associada a ITRS nas crianças.

Palavras-Chave: Tosse; Infecção do Trato Respiratório Superior; Crianças; Terapêutica.

\section{INTRODUÇÃO}

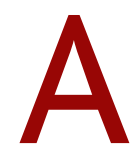
tosse é uma manobra expiratória explosiva, reflexa ou intencional, dirigida à limpeza do muco ou corpos estranhos da árvore traqueobrônquica. ${ }^{1,2}$ De acordo com a duração da sintomatologia a tosse pode ser dividida em aguda (duração inferior

* Interna de Medicina Geral e Familiar do Centro de Saúde da Senhora da Hora, Unidade Local de Saúde de Matosinhos. a três semanas), subaguda (presente por três a oito semanas) e crónica (presente para além das oito semanas). Qualquer que seja a duração este é um sintoma que merece ser correctamente investigado. ${ }^{3}$

Nas crianças a tosse aguda é um dos sintomas mais frequentemente associados às infecções do trato respiratório superior (ITRS). ${ }^{4,5} \mathrm{~A}$ tosse é um sintoma incómodo, não só porque preocupa os pais que ficam ansiosos com a possibilidade desta ser um sintoma de uma doen- 
ça grave, mas também porque pode incomodar o sono das crianças e dos próprios pais com a consequente diminuição do rendimento escolar e produtividade laboral. $^{6-8}$ É frequente que as crianças saudáveis desenvolvam cerca de cinco a oito ITRS por ano, cada uma das quais acompanhada por tosse, e mesmo aquelas sem ITRS podem tossir entre uma e trinta e quatro vezes por dia. ${ }^{7}$ Os infantários e escolas podem encorajar a que a criança com tosse permaneça em casa, o que condiciona mais dias de ausência ao trabalho e actividade lectivas. ${ }^{4}$

Sendo um sintoma tão comum, a tosse é um dos principais motivos de consulta nos cuidados de saúde primários. Alguns estudos internacionais referem que duas em cada três crianças consultam o seu médico de família com ITRS e cerca de três quartos destas têm tosse. ${ }^{5}$ Nos Estados Unidos este é o sintoma responsável por mais visitas ao médico de família. ${ }^{8}$ Os custos, com ela relacionados, para o Serviço Nacional de Saúde (SNS) do Reino Unido ascendem aos trinta e um milhões e meio de libras anuais, a maioria dos quais relacionados com a prestação de cuidados pelos médicos de Medicina Geral e Familiar. ${ }^{9}$ Em Portugal o último censo populacional de 2001 refere um total de 1656602 crianças (indivíduos com idade inferior ou igual a 15 anos), correspondendo a $16,0 \%$ da população total. ${ }^{10}$ Sendo assim, será de esperar que se realizem, pelo menos, 800000 consultas anuais relacionadas com a tosse, com os custos que isso representa para o SNS. Para além destes custos directos ainda há que contabilizar os custos com a medicação, nomeadamente com antibióticos muitas vezes desnecessários, e com medicação adquirida sem prescrição médica, que apesar de aprovada para uso a partir dos 6 anos, pode não só ser ineficaz, como pode ter também efeitos laterais perigosos. ${ }^{11-15}$

A incerteza relativamente à história natural da tosse em crianças pode ser parcialmente responsável pela alta taxa de consultas médicas e elevada prescrição de medicamentos. Há evidências que a partilha de informação e o aconselhamento são tácticas que permitem regular o acesso a consultas, diminuindo a procura de cuidados médicos ao mais pequeno sintoma. ${ }^{5}$

Tendo em conta que o médico de Medicina Geral e Familiar (MGF) é a «porta de entrada» do nosso SNS, será de esperar que seja a ele que os pais recorrem quando as suas crianças têm tosse e que este seja um motivo para uma elevada taxa de consultas. É por isso importante que o médico saiba lidar com este tipo de sintoma, munido da melhor evidência disponível, e saiba partilhar a informação com os pais para os tranquilizar e regular a procura de cuidados médicos.

É objectivo deste estudo efectuar uma revisão baseada na evidência acerca do tratamento da tosse associada às ITRS, em crianças com idades entre os seis e doze anos.

\section{MÉTODOS}

Foi realizada uma pesquisa nas bases de dados National Guideline Clearinghouse, Canadian Medical Association Infobase, Publeg, Cochrane Library, Dare, Trip Database, National Library of Guidelines Specialist Library e Pubmed, usando os termos MeSH child, cough, upper respiratory infection e therapeutic, e uma pesquisa no Índex das Revistas Médicas Portuguesas utilizando os DeCS tosse, infecção do trato respiratório superior, crianças e terapêutica, para obtenção de estudos escritos em Inglês, Espanhol, Francês ou Português englobando indivíduos de idades entre os seis e doze anos, publicados entre Janeiro de 1998 e Julho de 2008. Tendo em conta o reduzido número de estudos obtidos, a pesquisa foi entretanto alargada para incluir todos aqueles publicados antes desse período. Foram também revistas as listas de referências bibliográficas dos estudos relevantes.

Foram definidos como critérios de inclusão, estudos em crianças na faixa etária referida, saudáveis, com infecções agudas do trato respiratório superior, que comparassem a utilização de uma ou mais classes de fármacos para o tratamento da tosse com o placebo ou ausência de tratamento, e avaliassem os fármacos utilizados em relação à sua capacidade de diminuição do sintoma tosse (intensidade, frequência e número de despertares nocturnos devidos à tosse).

Os critérios de exclusão foram: estudos referentes à presença de tosse convulsa, tosse associada a infecções do trato respiratório inferior, tuberculose, bronquite crónica, asma e fibrose cística.

Foi utilizada a taxonomia Strenght of Recomendation Taxonomy (SORT) da American Family Physician ${ }^{16}$ para classificação dos artigos e atribuição de recomendações.

\section{RESULTADOS}

Foram identificados oitenta e dois estudos dos quais foram seleccionados doze que cumpriam os critérios de inclusão. Dos estudos seleccionados, três são ensaios clínicos aleatorizados controlados, quatro são revisões sistemáticas e cinco são normas de orientação clínica baseadas na evidência (NOC). 


\section{Anti-tússicos (Codeína e Dextrometorfano):}

Cinco estudos (duas revisões sistemáticas ${ }^{11,17}$ e três ensaios clínicos aleatorizados controlados ${ }^{8,18,19}$ ), com um total de 350 crianças participantes, analisaram a eficácia das substâncias anti-tússicas em comparação com placebo e outras substâncias usadas no tratamento da tosse. Todos os estudos referem que nem a Codeína nem o Dextrometorfano (DM) são mais eficazes que o placebo no tratamento da tosse em crianças - Nível de evidência 1.

Várias NOC baseadas na evidência ${ }^{4,6,14,20,21}$ analisaram a eficácia do uso de anti-tússicos no tratamento da tosse e concluíram pela inexistência de benefício na utilização destas substâncias - Força de Recomendação A.

\section{Expectorantes (Guaiafenesina, Bromexina, Carbocisteína e Acetilcisteína):}

Nesta revisão não foram encontrados estudos acerca do uso de expectorantes em crianças.

Anti-histamínicos e/ou Descongestionantes (Difenidramina, Clemastina, Clorfeniramina, Bromofeniramina/

/fenilpropanolamina, Bromofeniramina/fenilefrina/

/propanolamina)

Dois estudos, ${ }^{8,19}$ ambos ensaios clínicos aleatorizados controlados, com um total de 147 crianças participantes analisaram a eficácia do anti-histamínico Difenidramina na tosse nocturna em crianças, em comparação com DM e nenhum tratamento ou placebo, respectivamente. Ambos verificaram que não existem diferenças estatisticamente significativas entre os diferentes tipos de intervenções, e concluíram que os anti-histamínicos são comparáveis à ausência de tratamento e ao placebo no tratamento da tosse - Nível de evidência 2.

Os restantes anti-histamínicos (Clemastina e Clorfeniramina) e as combinações anti-histamínico/descongestionante acima referidos foram analisadas, em comparação com placebo, em duas revisões sistemáticas ${ }^{11,17}$ com um total de 394 participantes. Em ambos não foram encontradas diferenças estatisticamente significativas entre estes fármacos e o placebo na melhoria da tosse. Em ambos os estudos há a referir uma maior incidência de sonolência nas crianças tratadas com estes fármacos embora as diferenças também não sejam estatisticamente significativas - Nível de evidência 1.

Ressalta-se que apenas o anti-histamínico Difenidramina está disponível em Portugal em preparações para o tratamento da tosse.

\section{Metilxantinas:}

Nesta revisão não foram encontrados estudos acerca da utilização destas substâncias no tratamento da tosse associada a ITRS.

\section{Outras combinações de fármacos:}

Duas revisões sistemáticas ${ }^{11,17}$ avaliaram a eficácia de dois xaropes para a tosse pediátricos (Triaminicol ${ }^{\circledR}$ e Dorcol®) em comparação com placebo, num total de 43 crianças. Ambos os xaropes foram eficazes na diminuição da tosse, apesar da diferença relativamente ao placebo não ser estatisticamente significativa. - Nível de evidência 1. De referir que nenhuma destas preparações está disponível em Portugal.

A mesma revisão sistemática ${ }^{17}$ analisou um ensaio clínico aleatorizado e controlado envolvendo 51 crianças, comparando uma combinação de Dextrometorfano e Salbutamol com placebo, e chegou à conclusão que esta combinação não é mais eficaz que o placebo na diminuição da tosse. - Nível de evidência 1.

Uma revisão sistemática ${ }^{22}$ incluiu dois estudos aleatorizados controlados sobre o uso do $\beta$ - 2 agonista Albuterol em comparação com placebo, para o tratamento de crianças com tosse aguda sem evidência de obstrução brônquica. Nenhum dos estudos demonstrou benefícios com o uso desde fármaco e os resultados para a pontuação atribuída à tosse tenderam a piorar entre o dia 1 e 3 no grupo que recebeu Albuterol. - Nível de evidência 2.

Duas NOC baseadas na evidência ${ }^{6,14}$ analisaram o efeito da utilização dos $\beta$-2 agonistas Salbutamol e Albuterol no tratamento da tosse aguda, tendo ambas concluído que estes fármacos são ineficazes no tratamento deste sintoma - Força de Recomendação B.

\section{Hidratação oral:}

Uma revisão sistemática ${ }^{23}$ analisou o benefício do aumento da ingestão de líquidos no tratamento dos sintomas associados às infecções respiratórias. Os autores desta revisão não encontraram estudos aleatorizados e controlados que cumprissem os critérios de inclusão e concluíram que até à data, não existe evidência que permita recomendar a hidratação oral abundante-Nível de evidência 1.

\section{CONCLUSÕES}

Com esta revisão conclui-se que não há evidência científica na actualidade que permita recomendar qualquer 
terapêutica farmacológica para o tratamento da tosse associada a ITRS em crianças.

Não há evidência da eficácia do uso de anti-tússicos ou expectorantes para o tratamento da tosse em crianças 4,6,8,11,14,17-19,22,23 - Força de Recomendação A.

Não há evidência que o uso de anti-histamínicos para o tratamento da tosse em crianças seja benéfico e a ocorrência de efeitos laterais podem mesmo desencorajar a sua utilização $8,11,17,19$ - Força de Recomendação A.

Xaropes pediátricos ${ }^{11,17}$ para a tosse não parecem exercer um efeito estatisticamente significativo na diminuição da tosse e, dada a ausência de informação acerca das substâncias que os constituem, deverão ser evitados Força de Recomendação A.

Os $\beta$-2 agonistas (Salbutamol e Albuterol) não demonstraram benefícios no tratamento da tosse associada a ITRS nas crianças ${ }^{6,14,17,20}$ - Força de Recomendação B.

A hidratação abundante deve ser evitada uma vez que não existe evidência que esta possa ser benéfica ${ }^{21}$ - Força de Recomendação A.

As conclusões devem ser interpretadas com cuidado uma vez que o número de estudos em cada grupo de intervenções avaliado foi pequeno, a qualidade de alguns dos estudos é baixa, assim como são poucos os participantes em cada um. Os desenhos, as populações abrangidas, e os resultados dos mesmos variam significativamente. Frequentemente são permitidas outras medicações para além daquelas que servem para a base do estudo, muitos dos resultados previstos não são bem definidos, e há estudos que optam por comparar intervenções com a ausência de tratamento em vez de placebo. Por vezes, há alguma dificuldade em perceber concretamente os critérios de exclusão aplicados, o método de aleatorização e se os intervenientes são ou não cegos para a intervenção. Alguns estudos são patrocinados por companhias farmacêuticas, com as limitações que isso pode significar em termos de resultados, e outros há que não mencionam se há ou não conflitos de interesse.

Considerando tudo isto, seria importante a realização mais estudos aleatorizados controlados de boa qualidade, para determinar quais os tratamentos farmacológicos mais adequados para o tratamento da tosse associada a ITRS em crianças.

\section{REFERÊNCIAS BIBLIOGRAFICAS}

1. Porter RS, Kaplan JL, Homeier BP, Beers MH. The Merck Manual of Diagnosis and Therapy. $18^{\text {a }}$ edição. 2006.
2. Aranburu MI. Tos aguda. Em Boquet JE, Gómez JA e tal, editores. Guía de Actuación en Atención Primaria. Barcelona: Semfyc Ediciones; 2006. p. 181-188.

3. Pratter MR, Brightling CE, Boulet LP e Irwin RS. An Empiric Integrative Approach to the Management of Cough. Chest. 2006;129:222-231. Disponível em: URL: http://chestjournal.org/cgi/content/abstract/129/1_suppl/222S. [acedido em 02/05/2008].

4. Allen PJ, Kelley LK. Managing Acute Cough in Children - Evidence Based Guidelines. Pediatric Nursing. 2007;33(6):515-524.

5. Hay AD, Wilson AD. The Natural History of Acute Cough in Children aged 0-4 years in Primary Care: a systematic review. British Journal of General Practice. 2002;402-409.

6. Shields MD, Bush A, Everard ML, McKenzie S, Primhak R. British Thoracic Society Cough Guideline Group. Recommendations for the Assessment and Management of Cough in Children. Thorax. 2008:63;1-15;

Disponível em: URL: http://thorax.bmj.com/cgi/content/full/63/Suppl_3/iii1 [acedido em 02/05/2008].

7. Hay AD, Wilson A, Fahey T, Peters TJ. The Duration of Acute Cough in Pre-school Children Presenting to Primary Care: a Prospective Cohort Study. Family Practice. 2003:20(6);696-705.

8. Paul IM, Yoder KE, Crowell KR, Shaffer ML, McMillan HS, Carlson LC, et al. Effect of Dextromethorphan, Diphenhydramine, and Placebo on Nocturnal Cough and Sleep Quality for Coughing Children and their Parents. 2004:114(1);e85-90. Disponível em: URL: http://www.pediatrics.org/cgi/content/full/114/1/e85 [acedido em 02/05/2008].

9. Hollinghurst $\mathrm{S}$, Gorst $\mathrm{C}$, Fahey T, Hay AD. Measuring the Financial Burden of Acute Cough in Pre-school Children: a cost of illness study. BMC Family Practice. 2008: 9(10);1-6. Disponível em: URL: http://www.biomedcentral.com/1471-2296/9/10 [acedido em 10/05/2008].

10. Instituto Nacional de Estatística Disponível em: www.ine.pt [ acedido 05/06/2008].

11. Schroeder K, Fahey T. Should We Advise Parents to Administer Over the Counter Cough Medicines for Acute Cough? Systematic Review of Randomised Controlled Trials. Arch Dis Child. 2002:86;170-175.

12. Sharfstein JM, North M, Serwint JR. Over the Counter But No Longer Under the Radar - Pediatric Cough and Cold Medications. N Engl J Med. 2007:357(23); 2321-2324.

13. Gunn VL, Taha SH, Liebelt EL, Serwint JR. Toxicity of Over-the-Counter Cough and Cold Medications. Pediatrics. 2001:108;e52-57. Disponível em: URL: http://www.pediatrics.org/cgi/content/full/108/3/e52 [acedido em 10/05/2008)

14. American Academy of Pediatrics Committee on Drugs. Use of Codeine and Dextromethorphan - Cointaining Cough Remedies in Children. 1997:99(6); 918-920. Disponível em: URL: http://www.pediatrics.org/ cgi/content/full/99/6/918 [acedido em 10/05/2008].

15. Pediatric Toxicology Committee and Data Committee, National Association of Medical Examiners. Division of Healthcare Quality Promotion, National Center for Preparedness, Detection, and Control of Infectious Diseases. Srinivasan A, Budnitz D, Shehab N. Infant Deaths Associated with Cough and Cold Medications. Two States. 2005.

16. Ebell M, Siwek J, Weiss BD, Woolf SH, Susman J, Ewigman B, Bowman M. Strenght of Recomendation Taxonomy (SORT): A Patient Centered Approach to Grading Evidence in the Medical Literature. 
2004:69(3);548-556. Disponível em: URL: www.aafp.org/afp [acedido em 10/05/2008].

17. Schroeder K, Fahey T. Over-the-Counter Medications for Acute Cough in Children and Adults in Ambulatory Settings. Cochrane Database of Systematic Reviews. 2004:4;1-23.

18. Taylor JA, Novack AH, Almquist JR, Rogers JE. Efficacy of cough suppressants in children. Pediatric Pharmacology and Therapeutics. 1993:122;799-802.

19. Yoder KE, Shaffer ML, Tournous SJ, Paul IM. Child Assessment of Dextromethorphan, Diphenhydramine, and Placebo for Nocturnal Cough due to Upper Respiratory Infection. Clinical Pediatrics. 2006:45; 633640.

20. Irwin RS, Baumann MH, Bolser DC, Boulet $L P$, Braman SS, Brigtling $C E$, et al. Diagnosis and management of Cough Executive Summary: ACCP Evidence-Based Clinical Practice Guidelines. Chest. 2006:129;1-23. Disponível em: URL: http://chestjournal.org [acedido em 10/05/2008].

21. Bolser DC. Cough Supressant and Pharmacologic Protussive Therapy: ACCP Evidence-Based Clinical Practice Guidelines. Chest. 2006:129;238-249. Disponível em: URL: http://chestjournal.org [acedido em 10/05/2008].

22. Smucny JJ, Flynn CA, Becker LA, Glazier RH. Are $\beta$-2 agonists Effective Treatment for Acute Bronchitis or Acute Cough in Patients Without Underlying Pulmonary Disease? A Systematic Review. J Fam Pract. 2001: 50(11).
23. Guppy MPB, Mickan SM, Del Mar CB. Advising patients to increase fluid intake for treating acute respiratory infections. Cochrane Database of Systematic Reviews. 2005; 4:1-10.

A autora Marlene Sousa é editora associada da Revista Portuguesa de Clínica Geral e declara não ter estado envolvida no processo de decisão editorial para este artigo.

\section{AGRADECIMENTOS}

A autora deseja agradecer à sua orientadora de formação, Dra. Teresa Neto pelo apoio concedido na elaboração deste artigo e à Dra. Andreia Chaves Cerejo pela revisão do texto e por todas as críticas construtivas que the permitiram melhorar este trabalho.

\section{ENDEREÇO PARA CORRESPONDÊNCIA}

Marlene Sousa

Centro de Saúde Sra. da Hora

Rua da Lagoa, sem $n^{\circ}$

4460-352 Senhora da Hora

E-mail: marleneso@iol.pt

Recebido em 19/02/2009

Aceite para publicação em 16/04/2010

\section{ABSTRACT}

\section{THERAPEUTICS OF COUGH ASSOCIATED WITH UPPER RESPIRATORY TRACT INFECTIONS IN CHILDREN:WHAT IS THE BEST EVIDENCE?}

Objectives: Review the evidence about the therapeutics of cough associated with URTIs in children from six to twelve years of age.

Data Sources: The author s200earched the databases National Guideline Clearinghouse, Canadian Medical Association Infobase, Publeg, Cochrane Library, Dare, Trip Database, National Library of Guidelines Specialist Library, Pubmed and Índex das Revistas Médicas Portuguesas and the reference lists of relevant articles.

Review Methods: The author searched the databases listed using MeSH terms child, cough, upper respiratory infection, therapeutic, for all articles written in English, French, Spanish and Portuguese, published until July 2008. The studies were classified using Strenght of Recomendation Taxonomy (SORT). Studies excluded were those disagreeing with the objective of the study, those not referring to children and those including other diseases and conditions besides URTIs.

Results: Eighty two articles were collected and twelve were selected. From the studies analyzed, nine showed that antitussives, antihistamines and antihistamine/decongestant combinations aren't more effective than placebo for the treatment of cough. Another two, analyzed a combination of antitussive and a $\beta-2$ agonist and an isolated $\beta-2$ agonist, and found no improvement in the symptom, and another one didn't found benefit in increased fluid intake. Two studies showed a non-significant improvement of cough with the use of cough syrups. For expectorants and metilxanthines no studies were found.

Conclusion: There is no evidence for the use of antitussives, expectorants, antihistamines and increased oral fluid intake for the treatment of cough (SOR A). There is no evidence for the use of $\beta-2$ agonist in the treatment of this symptom (SOR B). Pediatric cough syrups have a non-significant effect in the treatment of cough. There is a need for high quality randomized controlled trials about the effectiveness of the drugs used in the treatment of cough associated with URTIs in children.

Keywords: Cough; Upper Respiratory Tract Infection; Children and Therapeutics. 